Research Article

\title{
Screening and Analysis of Key Genes in miRNA-mRNA Regulatory Network of Membranous Nephropathy
}

\author{
Yawei Hou, ${ }^{1}$ Yameng Li, ${ }^{1}$ Yichuan Wang, ${ }^{2}$ Wenpu $\mathrm{Li}^{3}$ and Zhenwei Xiao $\mathbb{D}^{3}$ \\ ${ }^{1}$ School of Traditional Chinese Medicine, Shandong University of Traditional Chinese Medicine, Jinan, China \\ ${ }^{2}$ Affiliated Hospital of Shandong University of Traditional Chinese Medicine Nephrology, Jinan, China \\ ${ }^{3}$ Shandong University of Traditional Chinese Medicine, Jinan, China \\ Correspondence should be addressed to Zhenwei Xiao; xiaozhenwei2021@163.com
}

Received 12 September 2021; Accepted 15 October 2021; Published 16 November 2021

Academic Editor: Kalidoss Rajakani

Copyright ( $\odot 2021$ Yawei Hou et al. This is an open access article distributed under the Creative Commons Attribution License, which permits unrestricted use, distribution, and reproduction in any medium, provided the original work is properly cited.

Background. MicroRNAs (miRNAs) are confirmed to participate in occurrence, development, and prevention of membranous nephropathy $(\mathrm{MN})$, but their mechanism of action is unclear. Objective. With the GEO database and the use of bioinformatics, miRNA-mRNA regulatory network genes relevant to $\mathrm{MN}$ were explored and their potential mechanism of action was explained. Methods. The MN-related miRNA chip data set (GSE51674) and mRNA chip data set (GSE108109) were downloaded from the GEO database. Differential analysis was performed using the GEO2R online tool. TargetScan, miRTarBase, and StarBase databases were used to predict potential downstream target genes regulated by differentially expressed miRNAs, and the intersection with differential genes were taken to obtain candidate target genes. According to the regulatory relationship between miRNA and mRNA, the miRNA-mRNA relationship pair was clarified and Cytoscape was used to construct a miRNA-mRNA regulatory network. WebGestalt was used to conduct enrichment analysis of the biological process of differential mRNAs in the regulatory network; FunRich analyzes the differential mRNA pathways in the miRNA-mRNA regulatory network. And the STRING database was used to construct a PPI network for candidate target genes, and Cytoscape visually analyzes the PPI network. Results. Experiments were conducted to screen differentially expressed miRNAs and mRNAs. There were 30 differentially expressed miRNAs, including 22 upregulated and 8 downregulated; and 1267 differentially expressed mRNAs, including 536 upregulated and 731 downregulated. Using TargetScan, miRTarBase, and StarBase databases to predict the downstream targets of differentially expressed miRNAs, 2957 downstream target genes coexisting in the 3 databases were predicted to intersect with differentially expressed mRNAs to obtain 175 candidate target genes. Finally, 36 miRNA-mRNA relationship pairs comprising 10 differentially expressed miRNAs and 27 differentially expressed mRNAs were screened out, and the regulatory network was constructed. Further analysis revealed that the miRNA regulatory network genes may be involved in the development of membranous nephropathy by mTOR, PDGFR- $\beta$, LKB1, and VEGF/VEGFR signaling pathways. Conclusion. The miRNA regulatory network genes may participate in the regulation of podocyte autophagy, lipid metabolism, and renal fibrosis through mTOR, PDGFR- $\beta$, LKB1, and VEGF/VEGFR signaling pathways, thereby affecting the occurrence and development of membranous nephropathy.

\section{Introduction}

Membranous nephropathy (MN) is a common pathological type of adult nephrotic syndrome. Its pathological manifestations are characterized by the formation of immune complexes under the epithelial cells of the outer visceral layer of the glomerular basement membrane and diffuse thickening of the glomerular basement membrane. The clinical manifestations are massive proteinuria, hypoalbuminemia, edema, and dyslipidemia. MN accounts for about $20 \%$ to $37 \%$ of adult nephrotic syndrome, and about $1 / 3$ of patients eventually develop end-stage renal disease [1]. $75 \%-80 \%$ of $\mathrm{MN}$ are idiopathic membranous nephropathy (IMN), and $20 \%-25 \%$ are secondary membranous nephropathy or atypical membranous nephropathy. The main causes of the latter are systemic lupus erythematosus, hepatitis B virus, use of NSAIDs, and malignant tumors [2]. Studies have shown a $13 \%$ increase in the risk of 
membranous nephropathy in China every year, which may be related to environmental pollution [3]. A single-center study in Beijing showed that, from 2003 to 2012, the incidence of IMN in primary glomerular disease increased from $16.8 \%$ to $29.35 \%$, and the proportion of young patients suffering from early primary membranous nephropathy increased significantly [4]. In the United States, the incidence of $\mathrm{MN}$ is estimated to be about 12 per million per year, with an average age between 50 and 60 years old, and the ratio of males to females is $2: 1$ [5-7]. In the United States, the incidence of ESRD caused by $\mathrm{MN}$ is about 1.9 per million per year [5]. In clinical observations, it was found that about $1 / 3$ of patients with primary membranous nephropathy will be completely relieved naturally, while another $1 / 3$ of patients will develop lifelong proteinuria while retaining kidney function for a long time, leaving $1 / 3$ of patients will progress to end-stage renal disease [8]. Membranous nephropathy seriously endangers human health and brings a heavy economic burden to individuals and society. Therefore, early detection of the disease, early diagnosis, and appropriate treatment play a vital role in preventing or delaying the deterioration of membranous nephropathy.

Renal biopsy is the gold standard for the diagnosis of membranous nephropathy. However, because it is a traumatic operation and has certain technical requirements for physicians, and renal biopsy cannot be performed in some patients due to various reasons, there are certain limitations in clinical applications. Therefore, exploring the potential regulatory mechanism of $\mathrm{MN}$ and identifying new potential biomarkers and drug target genes have important guiding significance for subsequent clinical diagnosis and treatment.

MicroRNA is a type of endogenous noncoding smallmolecule single-stranded RNA widely found in eukaryotes. It usually consists of 21-25 nucleotides and is highly conserved. It does not have an open reading frame itself. It participates in post-transcriptional gene regulation, affects the pathophysiological process of the body, and is related to cell development, differentiation, proliferation, apoptosis, immune regulation, tumorigenesis, etc. [9]. The study [10] found that, compared with the healthy group, the expression of miRNA-186 in the kidney tissue of patients with membranous nephropathy was significantly downregulated, and in vitro experiments proved that miRNA-186 via Toll-like receptor 4 (TLR4), P2X7, and caspase-3 participates in podocyte apoptosis, leading to increased basement membrane permeability, which in turn leads to membranous nephropathy. The study [9] found that, compared with healthy persons, increased levels of miRNA-193a were found in the urine of membranous nephropathy patients and are associated with an increase in urinary protein levels, thus increasing the severity of the disease. In addition, overexpression of miRNA-193a often indicates poor prognosis. However, there are few reports about the miRNA-miRNA regulatory network and the deep molecular mechanism of $\mathrm{MN}$, especially the miRNA-mediated regulatory mechanism and the molecular network involved in the prevention and treatment of $\mathrm{MN}$ are still unclear. Hence, the experiment intends to use the MN-related miRNA and mRNA expression data sets in the GEO database to construct a
miRNA-mRNA regulatory network using bioinformatics methods, screen key miRNA-mRNA regulatory relationship pairs, and analyze target functions and related signal pathways to explore their mechanism of action and provide important theoretical references and scientific basis for early diagnosis and targeted therapy of MN.

\section{Materials and Methods}

\subsection{Design. Molecular bioinformatics research.}

2.2. Time and Place. From July 2021 to August 2021, in the nephrologist's office of the Eastern District of Shandong University of Traditional Chinese Medicine Affiliated Hospital.

2.3. Data Source. The microarray data of miRNA expression profile and mRNA expression profile related to MN were retrieved from the GEO (Gene Expression Omnibus) database of NCBI (Table 1). Screening criteria: kidney samples from $\mathrm{MN}$ patients and healthy people are included, and cell lines or animal models are excluded. Finally, the miRNA expression data set GSE5167 and the mRNA expression data set GSE108109 that meet the requirements were downloaded. The data set GSE51674 was based on the platform GPL10656 and contained 16 kidney tissue samples, including 6 patients with membranous nephropathy, with an average age of 63.8 years, and 6 male patients; and there were 4 healthy patients with an average age of 38 years, 3 males and 1 female. The data set GSE108109 was based on the platform GPL19983 and contained 111 kidney tissue samples, including 6 healthy people and 44 membranous nephropathy patients.

\subsection{Methods}

2.4.1. Data Processing and Differential Expression Analysis. The online analysis tool GEO2R (https://www.ncbi.nlm.nih. gov/geo/geo2r/) from NCBI's GEO online analysis tool was used to obtain differential mRNAs and miRNAs and screen the differential genes. The standard setting is adj. $p$ value $<0.01$ and $\mid \log 2$ fold change (FC) $\mid>1$. The volcano map and cluster map are used to visually describe the differential expression data.

\subsubsection{Target Gene Prediction and miRNA-mRNA Regulatory} Network Construction. The target genes of differentially expressed miRNAs were predicted using the TargetScan [11], miRTarBase [12], and StarBase [13] databases. In order to obtain candidate target genes, search for the intersection of target genes predicted by all three databases and GSE108109 differential genes. According to the regulatory relationship between miRNA and mRNA, the miRNA-mRNA regulatory network is constructed. Cytoscape [14] software (version 3.7.2) was used for miRNA-mRNA regulatory network visualization. 
TABLE 1: Databases or software used in this study.

\begin{tabular}{lc}
\hline Database/software & URL \\
\hline GEO database & https://www.ncbi.nlm.nih.gov/geo/ \\
STRING database & https://string-db.org/ \\
TargetScan database & http://www.targetscan.org/vert_72 \\
miRTarBase database & https://mirtarbase.cuhk.edu.cn/ \\
StarBase database & https://starbase.sysu.edu.cn/ \\
WebGestalt database & http://www.webgestalt.org/ \\
FunRich database & http://www.funrich.org/ \\
STRING database & https://string-db.org/ \\
Cytoscape software (3.7.2) & https://cytoscape.org/ \\
\hline
\end{tabular}

2.4.3. Cross-Validation of External Data Sets. The MN-related mRNA expression data set GSE108113 was downloaded from the GEO database. GSE108113 was based on the platform GPL19983 and contained 280 kidney tissue samples, including 5 healthy patients and 87 patients with membranous nephropathy. In order to verify the common genes in the development of $\mathrm{MN}$, we look for the same differential mRNA in the datasets GSE108109 and GSE108113.

2.4.4. miRNA-Regulated Target Gene Function Enrichment and KEGG Pathway Analysis. The WebGestalt [15] online website was used to conduct biological process (BP) enrichment analysis of differential mRNAs in the regulatory network; FunRich [16] software was used to analyze the signal pathway of differential mRNA in the regulatory network.

2.4.5. Candidate Target Gene Protein-Protein Interaction Network Construction. In order to further identify the relationship between candidate target genes, the STRING database [17] is used for protein-protein interaction (PPI), and the Cytoscape software is used for visual analysis of the PPI network. The size of the node is represented by the degree value and is used by CytoHubba [18]. The plug-in MCC [18] algorithm screens out the top 20 core genes and takes the intersection with the target genes in the miRNA regulatory network.

\section{Results}

3.1. Differentially Expressed miRNA. Comparing the kidney tissue samples of patients with membranous nephropathy and healthy controls in the GSE51674 data set, 30 differentially expressed miRNAs were obtained, including 22 upregulated (hsa-miR-296-5p, hsa-miR-1249, hsa-miR1539, hsa-miR-602, hsa-miR-2116*, hsa-miR-210, hsa-miR106b, hsa-miR-222, hsa-miR-550, hsa-miR-17, hsa-miR-718, hsa-miR-660, hsa-miR-484, hsa-miR-532-5p, hsa-miR-17*, hsa-miR-503, hsa-miR-29c, hsa-miR-29a, hsa-miR-27b, hsamiR-26a, hsa-let-7g, and hsa-miR-24) and 8 downregulated (hsa-miR-29b-1*, hsa-miR-135a, hsa-miR-126*, hsa-miR125a-5p, hsa-miR-30c, hsa-miR-320d, hsa-miR-513a-5p, and hsa-miR-513b); a heat map and volcano map were plotted by using http://www.bioinformatics.com.cn, a free online platform for data analysis and visualization (Figure 1).
3.2. Differentially Expressed $m R N A$. Comparing the kidney tissues of patients with membranous nephropathy and healthy controls in the GSE108109 data set, 1267 differentially expressed mRNAs, including 536 upregulated expressions and 731 downregulated expressions, were obtained. Clustering of the top 50 differential mRNAs with a larger absolute value of the fold change is presented in Figures 2 and 3.

3.3. Target Gene Prediction and Regulatory Network Construction. TargetScan, miRTarBase, and StarBase databases were used to predict the downstream targets of differentially expressed miRNAs. Among them, 2957 mRNAs existed in the three databases at the same time (Figure 4(a)), and 2400 miRNA-mRNA relationship pairs existed in the 3 databases at the same time. The intersection of genes and differential genes that exist in the three databases was taken at the same time to obtain 175 candidate target genes (Figure 4(b) and Table 2).

According to the negative regulatory relationship between miRNA and mRNA, 36 miRNA-mRNA relationship pairs consisting of 10 differentially expressed miRNAs and 27 differentially expressed $\mathrm{mRNAs}$ were finally screened out. Cytoscape software was used to construct and visualize the miRNA-mRNA regulatory network (Figure 5 and Table 3 ).

3.4. Cross-Validation of External Data Sets. Using the same screening criteria (adj. $p$ value $<0.01,|\log 2 \mathrm{FC}|>1$ ), the GSE108113 data set was screened for differential genes; a total of 346 differential genes were screened, of which 121 were upregulated and 225 were downregulated. Compared with the GSE108109 data set, 44 differential genes were found to be upregulated at the same time, and 93 differential genes were downregulated at the same time (Figure 6).

3.5. Function Analysis of Network Target Genes. The BP function analysis of differential mRNAs in the regulatory network was carried out through the WebGestalt online website, and a total of 10 entries were enriched, 9 of which were statistically significant, mainly including cellular response to stress, positive regulation of nucleobase-containing compound metabolic process, apoptotic process, positive regulation of RNA metabolic process, response to steroid hormone, negative regulation of DNA biosynthetic process, muscle structure development, regulation of cellular response to stress, and response to organic cyclic compounds (Figure 7). FunRich software was used to analyze the differential mRNA pathways in the miRNA-mRNA regulatory network, which are mTOR, PDGFR- $\beta$, LKB1, and VEGF/VEGFR signaling pathways.

3.6. Construction of Differential Gene PPI Network. The downstream target genes existing in the three databases were intersected with the difference genes in the data set, 175 candidate target genes were obtained, the PPI network was constructed through the STRING database, and the software 


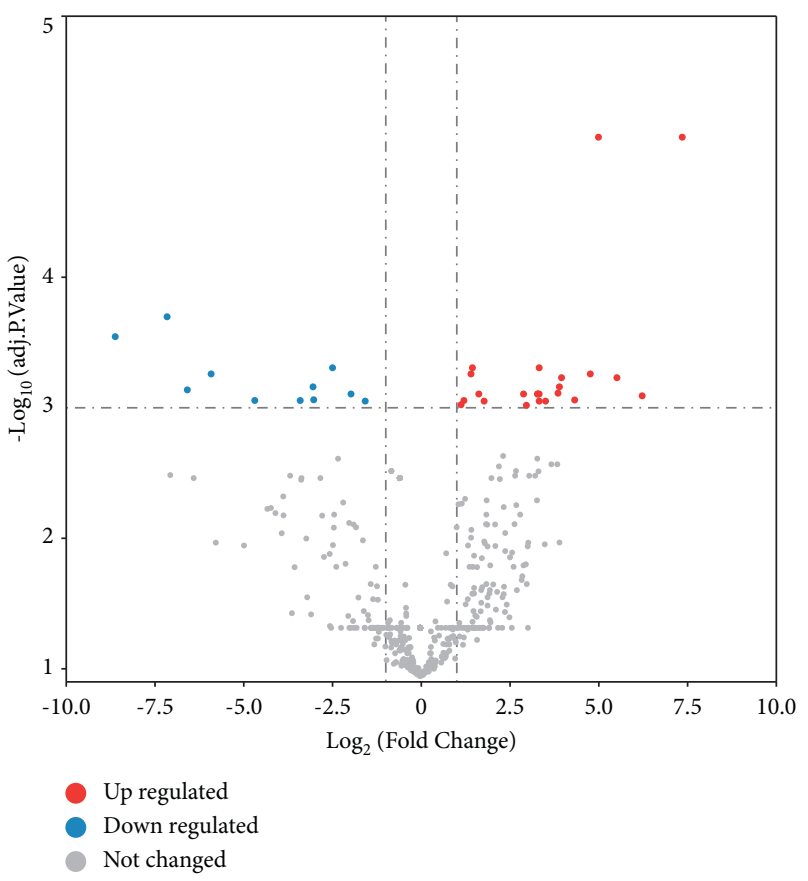

(a)

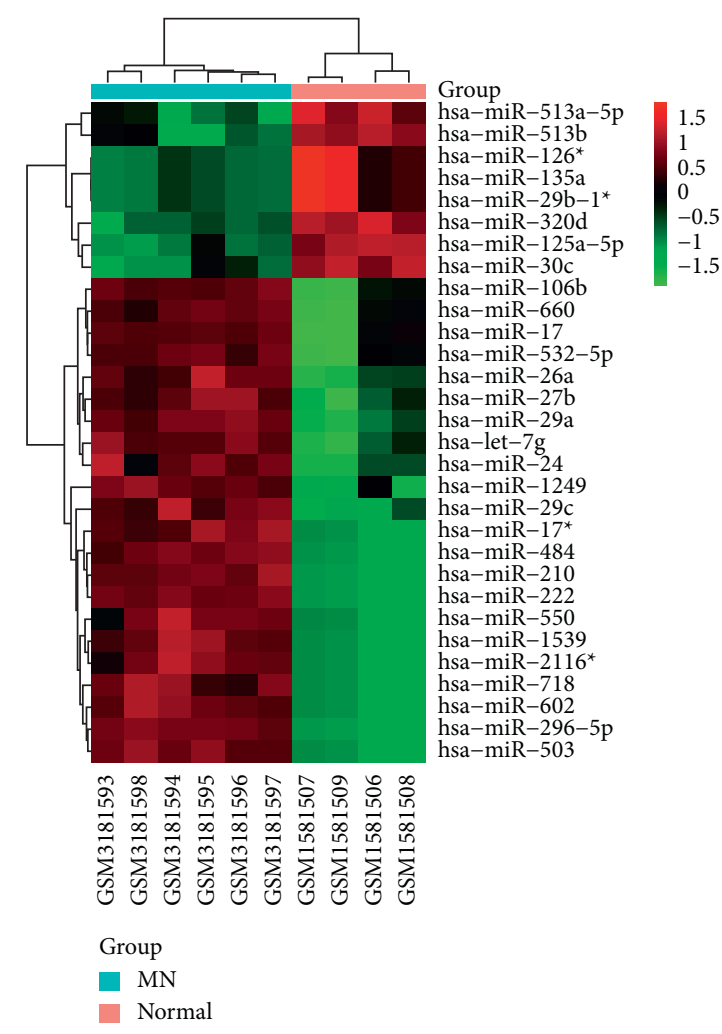

(b)

Figure 1: Differentially expressed miRNA kidney samples in patients with MN and healthy patients. (a) Volcano map of differentially expressed miRNAs. Red dots represent upregulation, blue dots represent downregulation, and gray dots represent no differential expression. (b) Heat map of differentially expressed miRNA. Red represents upregulation, and green represents downregulation.

Cytoscape was used to visually analyze the PPI network graph (Figure 8). The CytoHubba [18] plug-in MCC algorithm was used to screen out the first 20 hub genes (Figure 9(a)), and they were intersected with 27 differential genes in the miRNA-mRNA regulatory network, and finally, 3 hub target genes-NOTCH1, CCND2, and PIK3R1-were obtained (Figure 9(b)). Through the analysis of differentially expressed mRNAs, it is found that NOTCH1, CCND2, and PIK3R1 not only are core target genes but also exist in the miRNA-mRNA regulatory network. Studies have found that upregulation of NOTCH1 can aggravate the degree of renal interstitial fibrosis and the decline of glomerular filtration rate [19]. In addition, RNF152 and TET2 show significantly low expression in the kidney tissue of MN patients. Studies have shown that ring finger protein 152 (RNF152) prevents the activation of mTORC1 by targeting the small $G$ protein Rheb, thereby inhibiting the activity of the mTOR signaling pathway [20]. RNF152 can inhibit the activity of mTOR signaling pathway, thereby exerting renal protection. Previous studies have found that abnormal DNA methylation affects gene expression and disease development. Many studies have shown that the pathogenesis of nephrotic syndrome may be related to epigenetic changes [21, 22]. Studies have shown that [23] TET2 regulates DNA methylation and may participate in the occurrence and development of $\mathrm{MN}$ by regulating DNA methylation.

\section{Discussion}

With the development of gene sequencing technology and bioinformatics, the types of noncoding RNAs have been continuously improved, and their biological functions have also received increasing attention, which has become a current research hotspot in life sciences. The research on miRNAs has been gradually improved. miRNAs are widely expressed in various tissues and organs of the human body. One miRNA can regulate multiple target genes, and each target gene can be regulated by multiple miRNAs, thus forming a complex miRNA regulatory network. miRNA exists stably in blood, urine, and tissues, and blood and urine miRNAs can be detected without invasive procedures. And the expression in different kidney diseases is relatively specific, so miRNAs may develop into a new marker of MN in the future, which will help diagnose the disease early and evaluate the efficacy.

Although the pathogenesis of IMN is not yet clear, most scholars believe that IMN is an antibody-mediated autoimmune disease. The target antigen located in podocytes is recognized by autoantibodies and combined to form immune complexes deposited on basement membrane podocytes. Under the circumstances, activation of the complement system causes damage and shedding of podocytes, resulting in increased permeability of the 


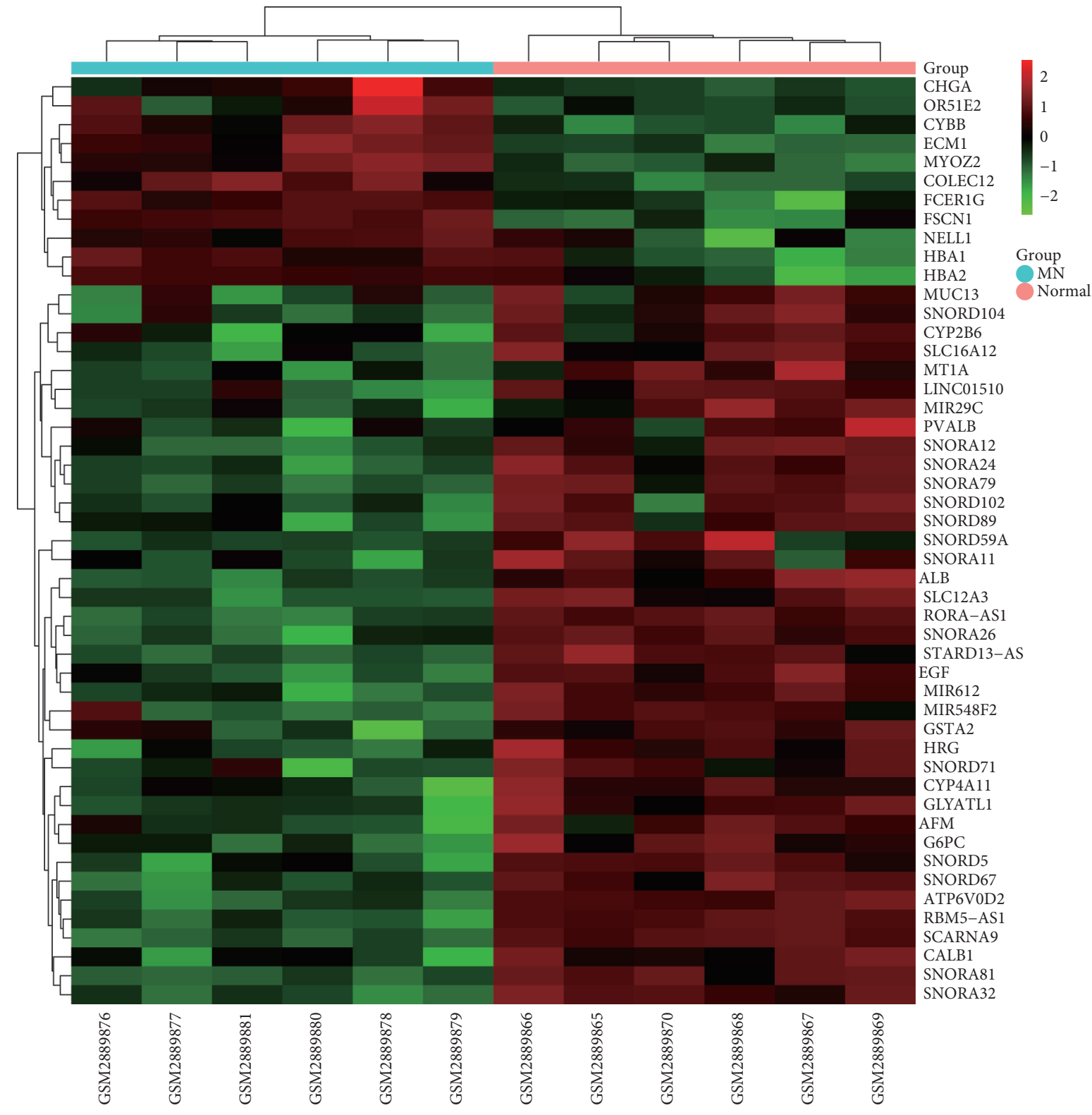

Figure 2: Heat map of the top 50 differentially expressed mRNAs with a larger absolute value of the fold change. Red represents upregulation, and green represents downregulation.

basement membrane and a large amount of proteinuria. Studies have shown that a large number of miRNAs have been confirmed to be closely related to the occurrence, mechanism, and prognosis of MN. For example, miRNA217 [24] promotes podocyte apoptosis by targeting tumor necrosis factor superfamily member 11 and then participates in the occurrence of MN; miRNA-328-5p [25] may participate in $\mathrm{MN}$ through inflammation and apoptosis-related pathways such as MAPK-related signaling pathways and p53 signaling pathways; miRNA-186 [10] via Toll-like receptor 4 (TLR4), P2X7, and cysteine caspase-3 participates in the apoptosis of podocytes, leading to increased permeability of the basement membrane, which in turn leads to membranous nephropathy; and miRNA-193a [9] may affect the occurrence of $\mathrm{MN}$ by influencing other related factors. Most of the above are focused on the upstream and downstream interactions between a single or several miRNAs/genes/ pathways, but the occurrence and development of diseases are the result of a multitarget, multipathway, and multistep synergistic effect. If you only study the relationship between a certain miRNA and gene, it will limit the study of the mechanism of $\mathrm{MN}$ to a certain extent.

In this experiment, 22 upregulated miRNAs and 8 downregulated miRNAs were excavated. TargetScan, miRTarBase, and StarBase databases were used to predict downstream targets of differentially expressed miRNAs. It is predicted that 2957 mRNAs exist in the three databases at the same time, and 2400 miRNA-mRNA relationship pairs exist in the 3 databases at the same time. Genes and differential genes that exist in the three databases were intersected at the same time to obtain 175 candidate target genes. According to the negative regulation relationship of 


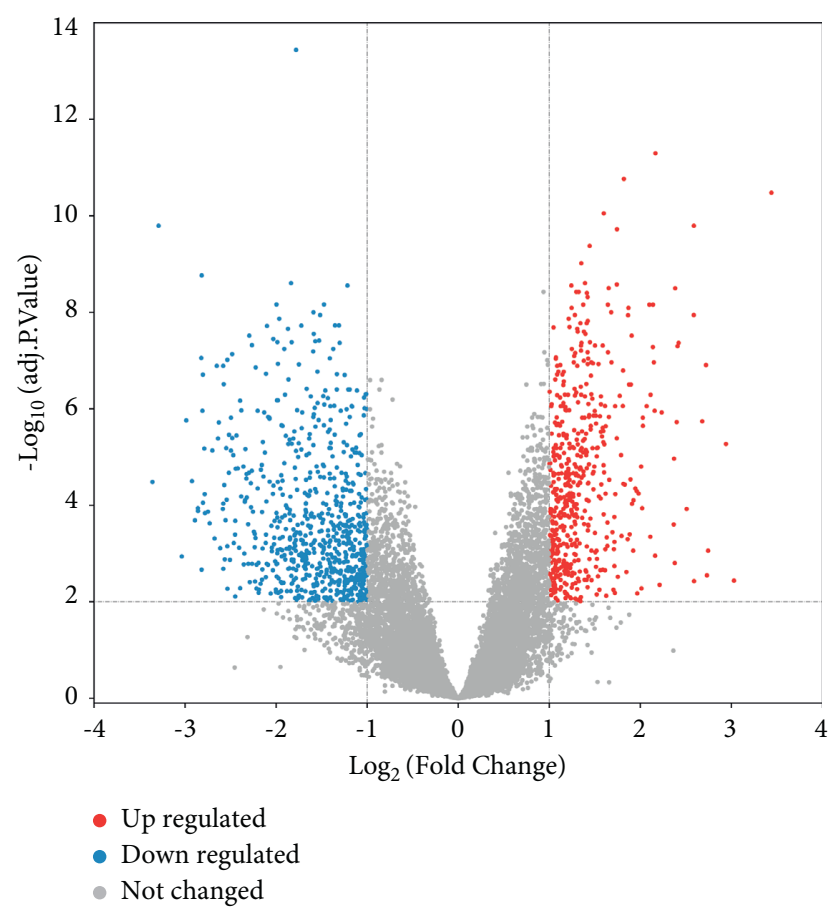

Figure 3: Volcano map of mRNAs differently expressed in MN. Red dots represent upregulation, blue dots represent downregulation, and gray dots represent no differential expression.

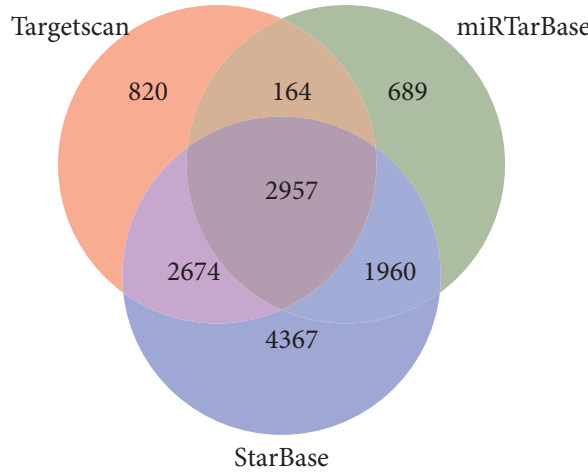

(a)

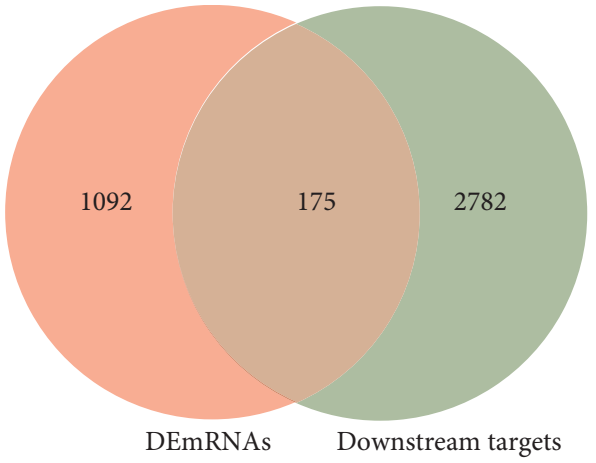

(b)

FIGURE 4: Screening of target genes. (a) Venn diagram of TargetScan, miRTarBase, and StarBase databases predicting miRNAs corresponding to downstream target genes. (b) Venn diagram of differentially expressed mRNA and miRNA downstream target genes.

miRNA and mRNA, 36 miRNA-mRNA relationship pairs comprising 10 differentially expressed miRNAs and 27 differentially expressed mRNAs are finally screened out. These differential miRNAs and mRNAs may be key nodes in the pathophysiology of $\mathrm{MN}$, and the first 20 core genes are screened out through the CytoHubba plug-in MCC algorithm, and they are intersected with 27 differential genes in the regulatory network to obtain the 3 genes NOTCH1, CCND2, and PIK3R1. Among them, NOTCH1 is significantly highly expressed in the kidney tissue of $\mathrm{MN}$ patients. Studies have shown that the degree of glomerular sclerosis and the urine protein level are positively correlated with the upregulation of NOTCH1 expression in renal podocytes, and the upregulation of NOTCH1 in patients with chronic renal failure can aggravate the degree of interstitial fibrosis and the decline of glomerular filtration rate [19].

Through FunRich analysis, it is found that the differentially expressed mRNAs in these regulatory networks are mainly related to mTOR, PDGFR- $\beta$, LKB1, and VEGF/ VEGFR signaling pathways, among which the mammalian target of rapamycin (mTOR) is a highly conserved serine/ threonine protein kinase, which is widely present in yeast to animal cells and belongs to the phosphatidylinositol-3-kinase-related kinase (PIKK) protein family [26]. Its stability affects the expression of cytokines in T cells; it participates in immunosuppression, affects transcription and protein synthesis, and regulates cell growth, apoptosis, and autophagy. Studies have shown that autophagy levels in $\mathrm{MN}$ 
TABLE 2: The intersection of mRNAs predicted by differentially expressed miRNAs and differentially expressed mRNAs in MN.

\begin{tabular}{|c|c|}
\hline mRNA & $\operatorname{LogFC}$ \\
\hline FSCN1 & 2.588635 \\
\hline SEMA7A & 2.421008 \\
\hline $\mathrm{ADM}$ & 2.409355 \\
\hline BMP2 & 2.383218 \\
\hline TNFRSF21 & 1.945833 \\
\hline NCAM1 & 1.925172 \\
\hline UCP2 & 1.909103 \\
\hline TGFB1 & 1.905343 \\
\hline EGR3 & 1.847593 \\
\hline BAMBI & 1.808341 \\
\hline CCND1 & 1.743998 \\
\hline CCDC85C & 1.655328 \\
\hline ITGA5 & 1.620048 \\
\hline COL1A2 & 1.615838 \\
\hline PLD3 & 1.599157 \\
\hline HBEGF & 1.597727 \\
\hline EHD2 & 1.593748 \\
\hline TRPC6 & 1.567959 \\
\hline GATA3 & 1.567876 \\
\hline TP53 & 1.541281 \\
\hline SKI & 1.532832 \\
\hline ST3GAL2 & 1.511352 \\
\hline PLEKHO1 & 1.502347 \\
\hline $\mathrm{HK} 2$ & 1.4997 \\
\hline NOTCH1 & 1.462259 \\
\hline TMEM154 & 1.430919 \\
\hline PDE4A & 1.430186 \\
\hline LYN & 1.424265 \\
\hline TMEM184B & 1.422096 \\
\hline PLXNC1 & 1.41303 \\
\hline KPNA2 & 1.40473 \\
\hline PREX1 & 1.401679 \\
\hline FAM129B & 1.387175 \\
\hline EIF5A2 & 1.383387 \\
\hline PXDN & 1.376657 \\
\hline DAG1 & 1.371781 \\
\hline FBRS & 1.361762 \\
\hline MTSS1L & 1.350619 \\
\hline KCTD12 & 1.34922 \\
\hline RNF44 & 1.343982 \\
\hline CENPP & 1.338335 \\
\hline PRR11 & 1.335654 \\
\hline VEGFC & 1.33485 \\
\hline PCDH17 & 1.333166 \\
\hline CD248 & 1.325592 \\
\hline LRP10 & 1.323391 \\
\hline CCND2 & 1.320625 \\
\hline ZNF703 & 1.31591 \\
\hline PLXND1 & 1.313283 \\
\hline RUSC2 & 1.312396 \\
\hline COL4A2 & 1.309219 \\
\hline COL4A1 & 1.307964 \\
\hline IER3 & 1.294667 \\
\hline SLIT3 & 1.29412 \\
\hline GNG2 & 1.290121 \\
\hline CBX6 & 1.275551 \\
\hline FKBP14 & 1.267006 \\
\hline ADAM12 & 1.255887 \\
\hline EMP1 & 1.248797 \\
\hline
\end{tabular}

TABLE 2: Continued.

\begin{tabular}{|c|c|}
\hline mRNA & LogFC \\
\hline PPM1F & 1.247126 \\
\hline ADAMTS1 & 1.243952 \\
\hline ZCCHC24 & 1.242563 \\
\hline SEMA4C & 1.234261 \\
\hline FN1 & 1.229458 \\
\hline MARK2 & 1.218718 \\
\hline SLC35E2 & 1.213068 \\
\hline ORAI1 & 1.209795 \\
\hline BTN2A2 & 1.202712 \\
\hline TMED9 & 1.201828 \\
\hline KIAA0930 & 1.200233 \\
\hline PIK3C2B & 1.199587 \\
\hline PRR12 & 1.189574 \\
\hline FICD & 1.188868 \\
\hline VASH1 & 1.18757 \\
\hline CDH5 & 1.179563 \\
\hline ANK1 & 1.175622 \\
\hline CDKN1C & 1.171323 \\
\hline MAFB & 1.160213 \\
\hline NETO2 & 1.158592 \\
\hline PACS1 & 1.155518 \\
\hline SLC7A1 & 1.12978 \\
\hline MAP1B & 1.123029 \\
\hline FMNL3 & 1.120955 \\
\hline NCOR2 & 1.118047 \\
\hline FOXK1 & 1.113134 \\
\hline VPS37B & 1.109738 \\
\hline SLC35C1 & 1.104002 \\
\hline CDC42SE1 & 1.10201 \\
\hline mRNA & $\log \mathrm{FC}$ \\
\hline IQSEC1 & 1.100248 \\
\hline C15orf39 & 1.099142 \\
\hline STK10 & 1.098527 \\
\hline MLXIP & 1.097227 \\
\hline PDGFRB & 1.094398 \\
\hline BCL7B & 1.092912 \\
\hline RRM2 & 1.083712 \\
\hline TNFAIP1 & 1.082132 \\
\hline RAB23 & 1.077515 \\
\hline TWIST1 & 1.077234 \\
\hline KIAA1211 & 1.075862 \\
\hline TNKS1BP1 & 1.075735 \\
\hline TRAM2 & 1.075199 \\
\hline ELK3 & 1.073763 \\
\hline BAG2 & 1.073373 \\
\hline BAK1 & 1.066222 \\
\hline ASB6 & 1.062721 \\
\hline HID1 & 1.062155 \\
\hline NFIX & 1.04586 \\
\hline PDGFB & 1.045391 \\
\hline SYNM & 1.043951 \\
\hline KMT2D & 1.042489 \\
\hline DTX3L & 1.033091 \\
\hline PGM2L1 & 1.029231 \\
\hline CDK6 & 1.025282 \\
\hline $\mathrm{DDN}$ & 1.022429 \\
\hline HYOU1 & 1.022252 \\
\hline GLTP & 1.022145 \\
\hline QSOX2 & 1.018591 \\
\hline ZNF598 & 1.017456 \\
\hline
\end{tabular}


TABle 2: Continued.

\begin{tabular}{|c|c|}
\hline mRNA & $\operatorname{LogFC}$ \\
\hline LRRC59 & 1.017138 \\
\hline TNRC18 & 1.014733 \\
\hline ANKRD52 & 1.011072 \\
\hline RAB1B & 1.002591 \\
\hline EFHD2 & 1.001892 \\
\hline DUSP1 & -1.00608 \\
\hline WNK3 & -1.00956 \\
\hline IRF2BP2 & -1.00999 \\
\hline SLC38A9 & -1.02822 \\
\hline ZFP36 & -1.03017 \\
\hline PIK3R1 & -1.0368 \\
\hline INTU & -1.04403 \\
\hline HNRNPC & -1.05119 \\
\hline TFRC & -1.07492 \\
\hline LRIG3 & -1.08372 \\
\hline MYSM1 & -1.08847 \\
\hline PAXBP1 & -1.09315 \\
\hline RORA & -1.09921 \\
\hline RND3 & -1.10045 \\
\hline IQCB1 & -1.1254 \\
\hline SCML1 & -1.13556 \\
\hline HNRNPA1 & -1.16882 \\
\hline CPM & -1.19419 \\
\hline ACADSB & -1.20615 \\
\hline NEDD9 & -1.20698 \\
\hline DDIT4 & -1.23395 \\
\hline DDX5 & -1.30958 \\
\hline CEBPD & -1.3324 \\
\hline ETNK2 & -1.33701 \\
\hline ANK3 & -1.34546 \\
\hline RDH10 & -1.35602 \\
\hline WSB1 & -1.35652 \\
\hline HLF & -1.3639 \\
\hline PFKFB2 & -1.36934 \\
\hline ANO3 & -1.37338 \\
\hline LUC7L2 & -1.40151 \\
\hline OGT & -1.41004 \\
\hline SLC16A9 & -1.45495 \\
\hline FKBP5 & -1.47532 \\
\hline $\mathrm{A} 1 \mathrm{CF}$ & -1.48213 \\
\hline RIMKLB & -1.51655 \\
\hline LUC7L3 & -1.52778 \\
\hline GATM & -1.53727 \\
\hline NABP1 & -1.5713 \\
\hline ALDH6A1 & -1.58547 \\
\hline TET2 & -1.59016 \\
\hline RNF152 & -1.6183 \\
\hline CHORDC1 & -1.67273 \\
\hline SLC4A4 & -1.67506 \\
\hline AGMAT & -1.68711 \\
\hline PRLR & -1.84312 \\
\hline IP6K3 & -1.90237 \\
\hline EYA4 & -1.91033 \\
\hline PDE7A & -1.93011 \\
\hline ANKS4B & -2.13921 \\
\hline GOLGA8A & -2.18853 \\
\hline GOLGA8B & -2.44931 \\
\hline
\end{tabular}

patients are abnormal. Animal experiments have found that podocyte autophagy in rats with membranous nephropathy is expressed at a high level, and there is a certain correlation between podocyte damage and shedding and autophagy. A number of studies have confirmed that autophagy is involved in the occurrence and development of $\mathrm{MN}[27,28]$. The mTOR signaling pathway may participate in the occurrence and development of $\mathrm{MN}$ by regulating the autophagy level of cells. The main function of platelet-derived growth factor (PDGF) is to regulate cell proliferation, $\mathrm{mi}-$ gration, inflammation, and tissue permeability and participate in extracellular matrix deposition. Chen et al. [29] found that blocking the PDGFR- $\beta$ signaling pathway in a rat model of chronic renal failure can inhibit the progression of renal fibrosis, and some patients with membranous nephropathy will also develop chronic renal failure. Blocking the PDGFR- $\beta$ signaling pathway may be beneficial to the long-term renal prognosis of $\mathrm{MN}$ patients. It provides a new treatment strategy for stabilizing the renal function of patients with MN. MN patients are mostly accompanied by dyslipidemia, and lipid metabolism disorders will promote the occurrence and development of the disease. Studies have found that [30] the AMPK-CaMKK $\beta$ (LKB1) signal transduction pathway plays a very important role in lipid metabolism. The LKB1 signaling pathway may affect MN by participating in lipid metabolism. At the same time, $\mathrm{MN}$ will eventually lead to renal fibrosis as the disease progresses. Studies have found that vascular endothelial growth factor (VEGF) is an important factor in maintaining the stability of the glomerular filtration barrier structure and the homeostasis of the kidney [31]. Experiments have shown that blocking the VEGF/VEGFR pathway can prevent the transformation of pericytes to myofibroblasts and then delay renal fibrosis [32].

Although the experiment constructed a potential miRNA-mRNA regulatory network based on bioinformatics, there are certain limitations. First of all, the number of kidney tissue cases in the healthy group is relatively small, which may affect the results of the experiment. The main sequencing method is chip sequencing. There is no high-throughput data set. In addition, there is a lack of data sets of the same population and the same platform. Second, the experimental results only involve membranous nephropathy kidney tissue specimens, not urine, blood, and other samples. Finally, the experiment only analyzed gene expression microarrays of membranous nephropathy and did not analyze RNA sequencing data, which lacked the ability to identify new features. In the future, more studies such as the dual luciferase report experiment will be designed to verify the in vivo and in vitro biological functions of the miRNA-mRNA regulatory network model.

In summary, based on the GEO chip data set, with the help of bioinformatics methods, 36 experimental miRNAmRNA regulatory relationship pairs related to $\mathrm{MN}$ were explored and the regulatory network was constructed to clarify the complex network of multiple targets and multiple pathways of $\mathrm{MN}$ regulation. The network core targets can improve the clinical performance of $\mathrm{MN}$ through $\mathrm{mTOR}$, PDGFR- $\beta$, LKB1, and VEGF/VEGFR signaling pathways and provide targets and reference directions for further indepth study of their mechanism of action and treatment of MN. 

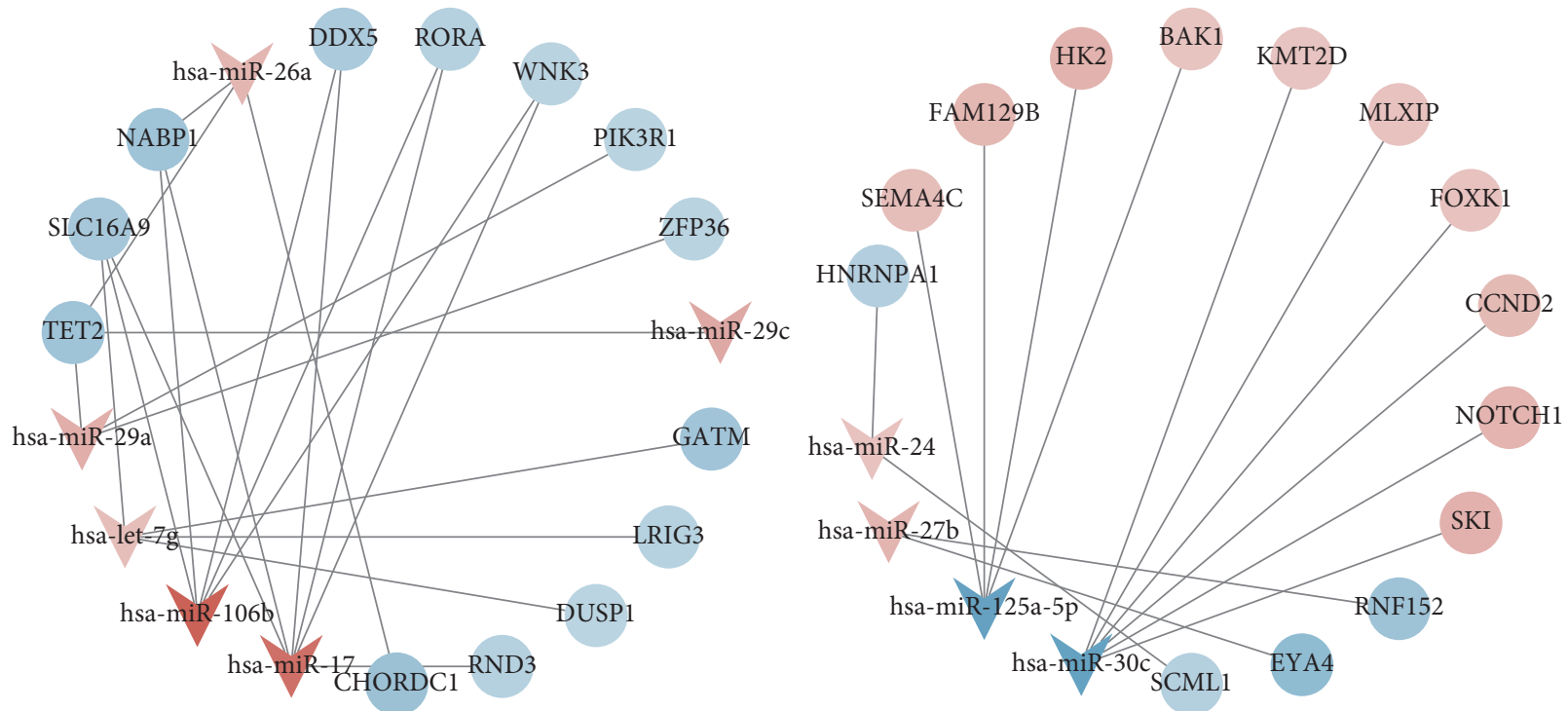

FIGURE 5: MN-related miRNA-mRNA regulatory networks. Red means the expression is upregulated, and blue means the expression is downregulated. miRNA and mRNA are represented by V-shape and circle, respectively.

TABLE 3: miRNA-mRNA regulatory pairs associated with MN.

\begin{tabular}{|c|c|c|c|}
\hline miRNA & Gene symbol & miRNA $\log F C$ & mRNA $\log F C$ \\
\hline hsa-let-7g & GATM & 1.2 & -1.53726793 \\
\hline hsa-let-7g & SLC16A9 & 1.2 & -1.45495451 \\
\hline hsa-let-7g & LRIG3 & 1.2 & -1.08372218 \\
\hline hsa-let-7g & DUSP1 & 1.2 & -1.00608428 \\
\hline hsa-miR-106b & NABP1 & 3.95 & -1.57129602 \\
\hline hsa-miR-106b & SLC16A9 & 3.95 & -1.45495451 \\
\hline hsa-miR-106b & DDX5 & 3.95 & -1.30958254 \\
\hline hsa-miR-106b & RORA & 3.95 & -1.09920587 \\
\hline hsa-miR-106b & WNK3 & 3.95 & -1.00956193 \\
\hline hsa-miR-125a5p & BAK1 & -3.03 & 1.06622167 \\
\hline hsa-miR-125a-5p & HK2 & -3.03 & 1.49970035 \\
\hline hsa-miR-125a-5p & FAM129B & -3.03 & 1.38717468 \\
\hline hsa-miR-125a-5p & SEMA4C & -3.03 & 1.2342607 \\
\hline hsa-miR-17 & NABP1 & 3.5 & -1.57129602 \\
\hline hsa-miR-17 & SLC16A9 & 3.5 & -1.45495451 \\
\hline hsa-miR-17 & DDX5 & 3.5 & -1.30958254 \\
\hline hsa-miR-17 & RND3 & 3.5 & -1.10045332 \\
\hline hsa-miR-17 & RORA & 3.5 & -1.09920587 \\
\hline hsa-miR-17 & WNK3 & 3.5 & -1.00956193 \\
\hline hsa-miR-24 & HNRNPA1 & 1.12 & -1.16881604 \\
\hline hsa-miR-24 & SCML1 & 1.12 & -1.13556225 \\
\hline hsa-miR-26a & CHORDC1 & 1.4 & -1.67272568 \\
\hline hsa-miR-26a & TET2 & 1.4 & -1.59015664 \\
\hline hsa-miR-26a & NABP1 & 1.4 & -1.57129602 \\
\hline hsa-miR-27b & EYA4 & 1.44 & -1.91033091 \\
\hline hsa-miR-27b & RNF152 & 1.44 & -1.61830472 \\
\hline hsa-miR-29a & TET2 & 1.62 & -1.59015664 \\
\hline hsa-miR-29a & PIK3R1 & 1.62 & -1.03679898 \\
\hline hsa-miR-29a & ZFP36 & 1.62 & -1.03016982 \\
\hline hsa-miR-29c & TET2 & 1.77 & -1.59015664 \\
\hline hsa-miR-30c & SKI & -3.05 & 1.5328315 \\
\hline hsa-miR-30c & NOTCH1 & -3.05 & 1.46225942 \\
\hline hsa-miR-30c & CCND2 & -3.05 & 1.32062533 \\
\hline hsa-miR-30c & FOXK1 & -3.05 & 1.1131342 \\
\hline hsa-miR-30c & MLXIP & -3.05 & 1.09722718 \\
\hline hsa-miR-30c & KMT2D & -3.05 & 1.04248918 \\
\hline
\end{tabular}




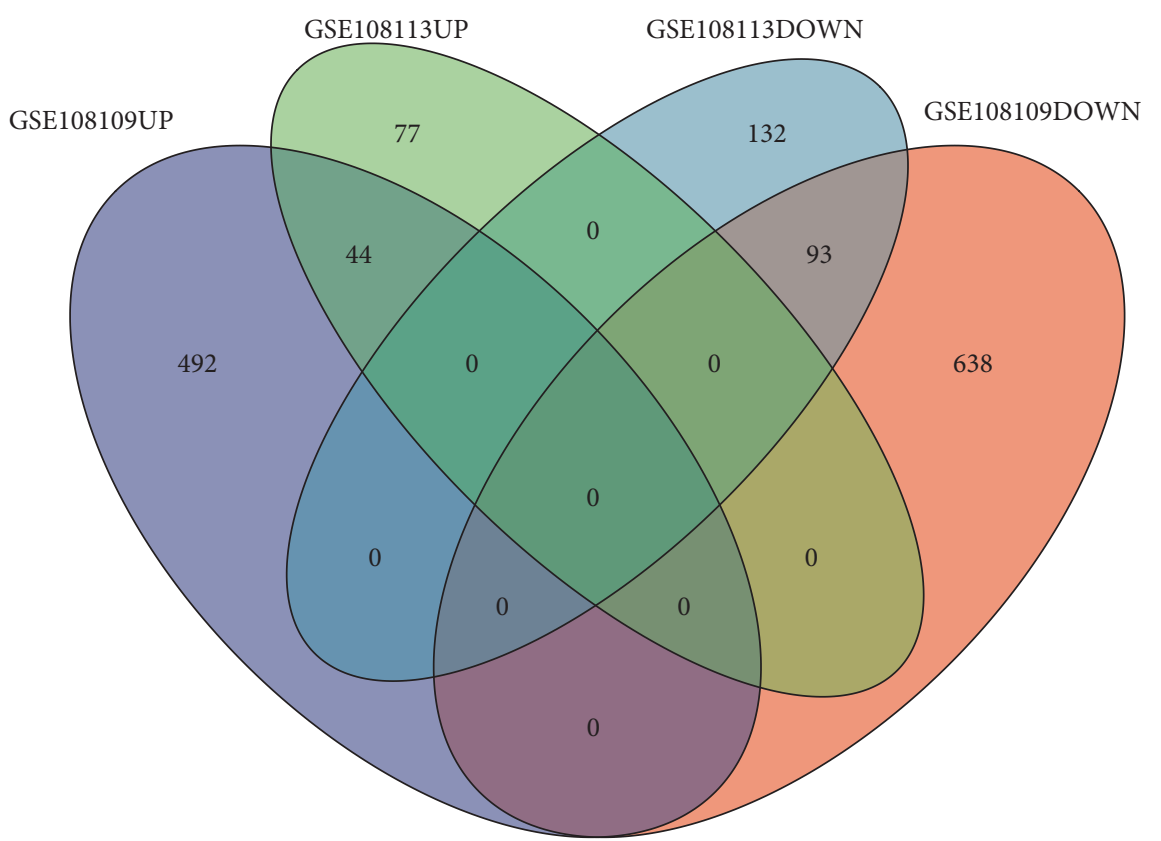

Figure 6: External data set cross-validation Venn diagram.

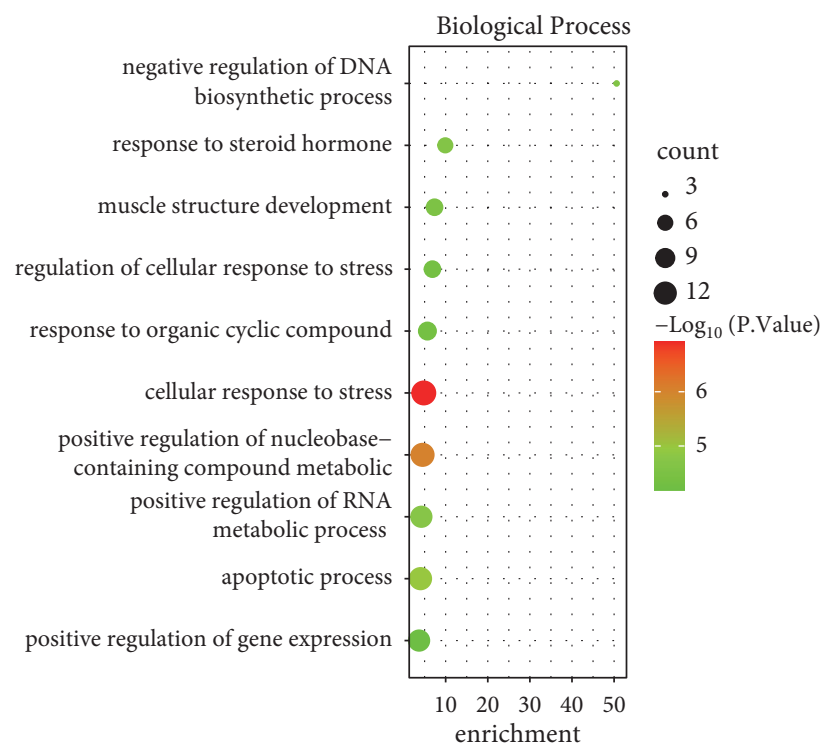

FIGURE 7: BP enrichment entries of 27 target genes in the miRNA-mRNA regulatory network. 


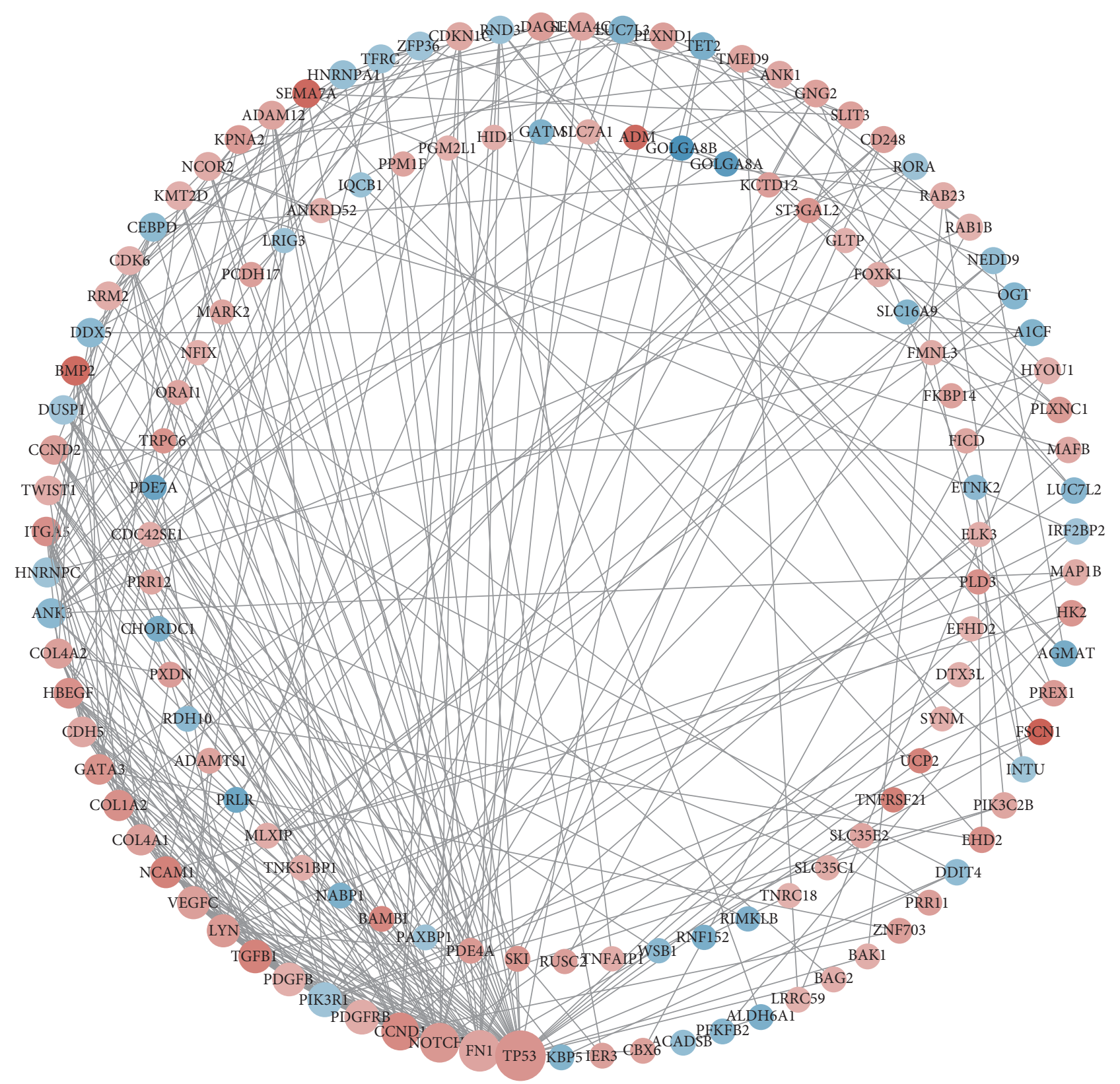

FIGURE 8: PPI network of candidate target genes: red dots represent upregulation, blue dots represent downregulation, and the size of the graph is determined by the degree value. 


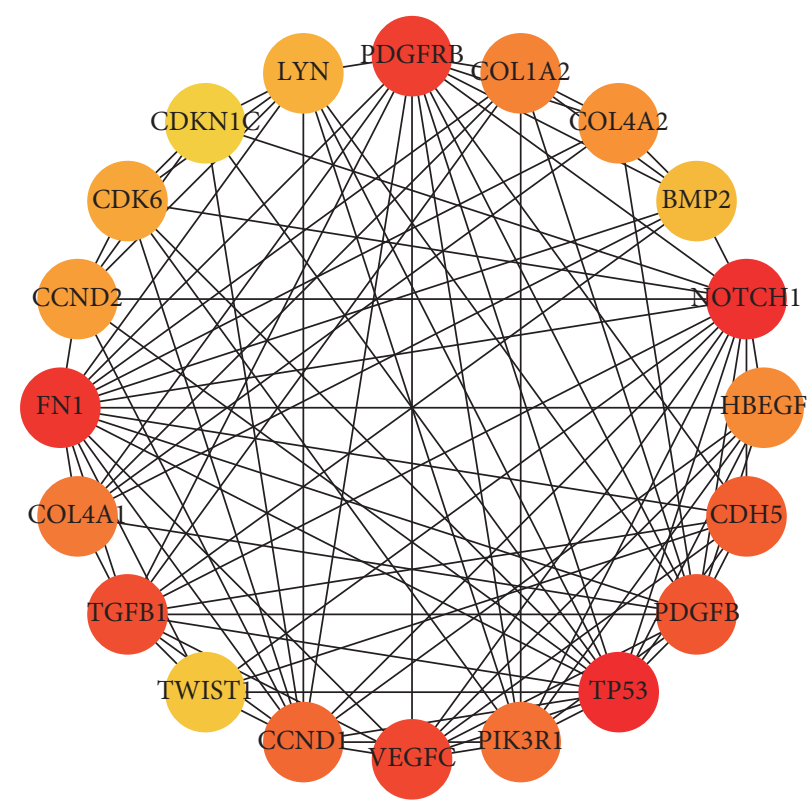

(a)

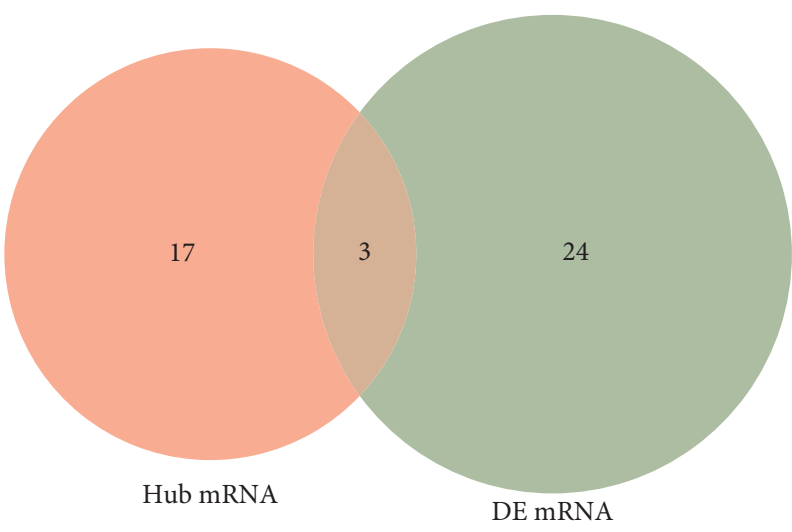

(b)

Figure 9: Hub genes and hub target genes. (a) The first 20 hub genes. (b) The first 20 hub genes and they were intersected with 27 differential genes in the miRNA-mRNA regulatory network.

\section{Data Availability}

The simulation experiment data used to support the findings of this study are available from the corresponding author upon request.

\section{Conflicts of Interest}

The authors declare that there are no conflicts of interest regarding the publication of this paper.

\section{Authors' Contributions}

The subject/experiment design was developed by $\mathrm{YH}$ and $\mathrm{ZX}$, and the experiment was carried out by YH, YL, and YW. Experimental evaluation was performed by $\mathrm{YW}$ and ZX. Data collection was done by YH, YL, and WL.

\section{Acknowledgments}

The authors thank Dr. Xuezhen Liang for his assistance in the experiment and his valuable comments.

\section{References}

[1] W. G. Couser, "Primary membranous nephropathy," Clinical Journal of the American Society of Nephrology, vol. 12, no. 6, pp. 983-997, 2017.

[2] J. K.-C. Ng, T. K.-W. Ma, F. M.-M. Lai et al., "Causes of nephrotic syndrome and nephrotic-range proteinuria are different in adult Chinese patients: a single centre study over 33 years," Nephrology, vol. 23, no. 6, pp. 565-572, 2018.

[3] X. Xu, G. Wang, N. Chen et al., "Long-term exposure to air pollution and increased risk of membranous nephropathy in
China," Journal of the American Society of Nephrology, vol. 27, no. 12, pp. 3739-3746, 2016.

[4] P. Zhu, F.-D. Zhou, S.-X. Wang, M.-H. Zhao, and H.-Y. Wang, "Increasing frequency of idiopathic membranous nephropathy in primary glomerular disease: a 10 -year renal biopsy study from a single Chinese nephrology centre," Nephrology, vol. 20, no. 8, pp. 560-566, 2015.

[5] D. C. Cattran and P. E. Brenchley, "Membranous nephropathy: integrating basic science into improved clinical management," Kidney International, vol. 91, no. 3, pp. 566-574, 2017.

[6] V. Kumar, R. Ramachandran, A. Kumar et al., "Antibodies to m-type phospholipase A2 receptor in children with idiopathic membranous nephropathy," Nephrology, vol. 20, no. 8, pp. 572-575, 2015.

[7] A. S. De Vriese, R. J. Glassock, K. A. Nath, S. Sethi, and F. C. Fervenza, "A proposal for a serology-based approach to membranous nephropathy," Journal of the American Society of Nephrology, vol. 28, no. 2, pp. 421-430, 2017.

[8] S. Ren, Y. Wang, L. Xian et al., "Comparative effectiveness and tolerance of immunosuppressive treatments for idiopathic membranous nephropathy: a network meta-analysis," PLoS One, vol. 12, no. 9, Article ID e0184398, 2017.

[9] M. Nalewajska, K. Gurazda, E. Styczyńska-Kowalska, M Marchelek-Myśliwiec, A Pawlik, and V Dziedziejko, "The role of MicroRNAs in selected forms of glomerulonephritis," International Journal of Molecular Sciences, vol. 20, no. 20, 2019.

[10] W.-G. Sha, L. Shen, L. Zhou, D.-Y. Xu, and G.-Y. Lu, "Downregulation of miR-186 contributes to podocytes apoptosis in membranous nephropathy," Biomedicine \& Pharmacotherapy, vol. 75, pp. 179-184, 2015.

[11] B. Fromm, T. Billipp, L. E. Peck et al., "A uniform system for the annotation of vertebrate microRNA genes and the evolution of the human microRNAome," Annual Review of Genetics, vol. 49, no. 1, pp. 213-242, 2015. 
[12] C.-H. Chou, N.-W. Chang, S. Shrestha et al., "miRTarBase 2016: updates to the experimentally validated miRNA-target interactions database," Nucleic Acids Research, vol. 44, no. D1, pp. D239-D247, 2016.

[13] J. H. Li, S. Liu, H. Zhou, L. H Qu, and J. H Yang, "starBase v2.0: decoding miRNA-ceRNA, miRNA-ncRNA and proteinRNA interaction networks from large-scale CLIP-Seq data," Nucleic Acids Research, vol. 42, no. Database issue, pp. D92-D97, 2014.

[14] P. Shannon, A. Markiel, O. Ozier et al., "Cytoscape: a software environment for integrated models of biomolecular interaction networks," Genome Research, vol. 13, no. 11, pp. 2498-2504, 2003.

[15] Y. Liao, J. Wang, E. J. Jaehnig, Z. Shi, and B. Zhang, "WebGestalt 2019: gene set analysis toolkit with revamped UIs and APIs," Nucleic Acids Research, vol. 47, no. W1, pp. W199-w205, 2019.

[16] M. Pathan, S. Keerthikumar, C.-S. Ang et al., "FunRich: an open access standalone functional enrichment and interaction network analysis tool," Proteomics, vol. 15, no. 15, pp. 2597-2601, 2015.

[17] D. Szklarczyk, A. L. Gable, K. C. Nastou et al., "The STRING database in 2021: customizable protein-protein networks, and functional characterization of user-uploaded gene/measurement sets," Nucleic Acids Research, vol. 49, no. D1, pp. D605-D612, 2021.

[18] C. H. Chin, S. H. Chen, H. H. Wu, and C. Ho, "Cytohubba: identifying hub objects and sub-networks from complex interactome," BMC Systems Biology, vol. 8, no. Suppl 4, p. S11, 2014.

[19] M. Murea, J.-K. Park, S. Sharma et al., "Expression of Notch pathway proteins correlates with albuminuria, glomerulosclerosis, and renal function," Kidney International, vol. 78, no. 5, pp. 514-522, 2010.

[20] L. Deng, L. Chen, L. Zhao et al., "Ubiquitination of Rheb governs growth factor-induced mTORC1 activation," Cell Research, vol. 29, no. 2, pp. 136-150, 2019.

[21] Y. Kobayashi, A. Aizawa, T. Takizawa, K. Igarashi, I. Hatada, and H. Arakawa, "Changes in DNA methylation in naïve T helper cells regulate the pathophysiological state in minimalchange nephrotic syndrome," BMC Research Notes, vol. 10, no. 1, p. 480, 2017.

[22] Y. Kobayashi, A. Aizawa, T. Takizawa et al., "DNA methylation changes between relapse and remission of minimal change nephrotic syndrome," Pediatric Nephrology, vol. 27, no. 12, pp. 2233-2241, 2012.

[23] H. Wu and Y. Zhang, "Reversing DNA methylation: mechanisms, genomics, and biological functions," Cell, vol. 156, no. 1-2, pp. 45-68, 2014.

[24] J. Li, B. Liu, H. Xue, Q. Q Zhou, and L Peng, "miR-217 is a useful diagnostic biomarker and regulates human podocyte cells apoptosis via targeting TNFSF11 in membranous nephropathy," BioMed Research International, vol. 2017, Article ID 2168767, 2017.

[25] G. Zhou, X. Zhang, W. Wang, W. Zhang, H. Wang, and G. Xin, "Both peripheral blood and urinary miR-195-5p, miR192-3p, miR-328-5p and their target genes PPM1A, RAB1A and BRSK1 may be potential biomarkers for membranous nephropathy," Medical Science Monitor, vol. 25, pp. 19031916, 2019.

[26] S. H. Hare and A. J. Harvey, "mTOR function and therapeutic targeting in breast cancer," American journal of cancer research, vol. 7, no. 3, pp. 383-404, 2017.
[27] S. Liang, J. Jin, J. Gong, B. Lin, Y. Li, and Q. He, "How many podocyte autophagosomes are there in immunoglobulin A nephropathy and idiopathic membranous nephropathy?" International Urology and Nephrology, vol. 48, no. 12, pp. 2109-2114, 2016.

[28] J. Jin, H. Zhan, B. Lin, Y. Li, W. Zhang, and Q. He, "Association of podocyte autophagosome numbers with idiopathic membranous nephropathy and secondary membranous nephropathy," International Urology and Nephrology, vol. 49, no. 6, pp. 1025-1031, 2017.

[29] Y.-T. Chen, F.-C. Chang, C.-F. Wu et al., "Platelet-derived growth factor receptor signaling activates pericyte-myofibroblast transition in obstructive and post-ischemic kidney fibrosis," Kidney International, vol. 80, no. 11, pp. 1170-1181, 2011.

[30] M. S. Seo, J. H. Kim, H. J. Kim, K. C. Chang, and S. W. Park, "Honokiol activates the LKB1-AMPK signaling pathway and attenuates the lipid accumulation in hepatocytes," Toxicology and Applied Pharmacology, vol. 284, no. 2, pp. 113-124, 2015.

[31] F. C. Brosius and R. J. Coward, "Podocytes, signaling pathways, and vascular factors in diabetic kidney disease," Advances in Chronic Kidney Disease, vol. 21, no. 3, pp. 304-310, 2014.

[32] S. Hakroush, M. J. Moeller, F. Theilig et al., "Effects of increased renal tubular vascular endothelial growth factor (VEGF) on fibrosis, cyst formation, and glomerular disease," American Journal Of Pathology, vol. 175, no. 5, pp. 1883-1895, 2009. 